\title{
Relationship between LA Strain by 2D Speckle Tracking and LV Diastolic Function in Patient with Different Grades of LV Diastolic Dysfunction
}

Wael Mohammed Attia, Alsayed Ali Abdou Almarghany, Mahmoud Abd El salam Ahmed

Department of Cardiology, Faculty of Medicine, Al-Azhar University, Cairo, Egypt

*Corresponding author: Mahmoud Abd El Salam, Email: mahmoud_cardio5800@yahoo.com; Mobile: (+20) 1012859078

\begin{abstract}
Background: Diastolic function assessment is complex and multipara metric because most conventional parameters do not follow the progression of diastolic dysfunction (DD). Strain imaging is an emerging index of LA function, with recent data demonstrating that LA strain is diminished in diastolic heart failure. However, LA strain is not part of the standard assessment of diastolic function. We hypothesized that LA strain decreases with worsening DD in a stepwise fashion and could thus be useful in evaluating DD.

Objectives: The aim of the current work was to observe the relationship between left atrial (LA) strain and left ventricular diastolic function. In addition to determine whether LA strain could be used to detect diastolic dysfunction (DD) and classify its degree when present. Patients and Methods: This prospective study included a total of 60 patients with diastolic dysfunction and 20 healthy control individuals, attending at outpatient clinic and Echocardiography Laboratory, Cardiology Department, Al-Hussein University Hospital. They were subjected to detailed echocardiographic examinations of the LV systolic and diastolic function and Lt atrial strain by 2D speckle tracking.

Results: There were statistically highly significant differences between the four groups as regard results of GLS strain $(\mathrm{P}$ value $=0.001)$ and peak LA strain $(\mathrm{P}$ value $=0.001)$.

Conclusions: It could be concluded that LA strain measurements are feasible and allow accurate categorization of DD, because unlike the traditional parameters, it changes progressively with severity of DD. LA strain may become a useful tool for diastolic assessment in future clinical practice.
\end{abstract}

Keywords: Two-Dimensional Speckle Tracking Echocardiography, Left atrial strain, Diastolic Dysfunction.

\section{INTRODUCTION}

The principal role of the left atrium (LA) is to modulate left ventricular (LV) filling via its reservoir, conduit, and booster functions. The LA manifests adaptive changes in its structure and mechanics, and these changes are well described in the setting of abnormal patterns of LV filling, known as diastolic dysfunction (DD). The use of Doppler echocardiography is a cornerstone for the diagnosis and categorization of diastolic function. Although there are already well-established algorithms available for DD staging that are widely used in the clinical setting, the criteria required for diagnosis incorporate multiple echocardiography-based parameters and can be cumbersome to acquire and interpret. Furthermore, individual patients may demonstrate a spectrum of diastolic indices that do not clearly meet the strict definition of a particular DD type, thereby making interpretation challenging at times ${ }^{(1,2)}$.

Strain imaging using 2-dimensional speckle tracking of the LA has been used for the assessment of left atrial function ${ }^{(3,4)}$. LA strain is angle-independent, and thus less susceptible to the limitations of Doppler echocardiographic assessment of strain ${ }^{(5)}$. Alterations in LA strain have been described in patients with hypertension, atrial fibrillation and diastolic heart failure (HF) ${ }^{(6-8)}$. However, there is limited data describing the changes in LA strain across DD groups, nor are there available thresholds proposed for use in the clinical evaluation of diastolic function. We hypothesized that LA strain measurements may hold promise as a simple noninvasive tool to aid in the determination of DD severity. The aim of this study was to determine whether LA strain could be used as an accurate diagnostic criterion for the presence and degree of DD, when compared with the current standard guidelines. Independent group of patients (validation cohort) and compared with the diagnosis conferred by guideline-based DD assessment.

\section{AIM OF THE WORK}

The aim of the current work was to observe the relationship between left atrial (LA) strain and left ventricular diastolic function. In addition to determine whether LA strain could be used to detect diastolic dysfunction (DD) and classify its degree when present.

\section{SUBJECTS AND METHODS}

This prospective study included a total of 60 patients with diastolic dysfunction and 20 healthy control individuals (normal ventricular function and ECG, and no cardiac risk factors), attending at outpatient clinic and Echocardiography Laboratory, Cardiology Department, Al-Hussein University Hospital. This study was conducted between .

Ethical approval and written informed consent: The study was approved by the Medical Ethics Committee of Al-Azhar University and a written informed consent was obtained from all patients. 
The included subjects were classified into control group in addition to three patient groups with different grades of diastolic dysfunction as the following:

Group 1: Healthy control group (20) subjects.

Group 2: Grade I diastolic dysfunction (20) patients.

Group 3: Grade II diastolic dysfunction (20) patients.

Group 4: Grade III diastolic dysfunction (20) patients.

We have performed a prospective study to derive and test LA strain thresholds for diastolic dysfunction in patients with preserved left ventricular ejection fraction.

Two-dimensional speckle tracking was used to measure peak LA strain, which was applied as a single parameter to classify diastolic dysfunction.

American Society of Echocardiography guidelines were used as the reference standard.

\section{Inclusion criteria:}

- The patient with LV ejection fraction $(\mathrm{EF}) \geq 50 \%$,

- Normal sinus rhythm

- No significant valvular heart disease (defined as greater than mild regurgitation or stenosis) or a prosthetic valve.

\section{Exclusion criteria:}

- Refusal of the patients to participate in the study.

- If images were of poor quality.

- If image loops did not depict all LA segments, did not allow speckle tracking of atrial boundaries $(<15 \%$ of the patients), which might preclude accurate strain measurements.

Careful history was taken from all the subjects with special emphasis on:

1. History of hypertension ${ }^{(9)}$, diabetes mellitus, ischemic heart disease (UA, MI, PTCA, CABG etc.) and smoking (9).

2. General and local cardiac examination was done for all subjects including vital signs with Jugular Venous Pressure (JVP), cardiac examination which involved precordial examination to detect clinically heart size, heart sounds, added sound, murmurs and examination of the back). Other systems will also be examined relevant systems were also examined, Respiratory and Musculoskeletal systems.

3. Resting surface 12 leads ECG was done for all subjects searching for rate, rhythm, BBB and chamber enlargement and ischemic changes ${ }^{(\mathbf{1 0})}$.

\section{Echocardiography:}

- All subjects were examined at rest in the left lateral decubitus position to obtain adequate images in different standard views. Standard 2D TTE examination were performed with a "Philips iE33 X Matrix" ultrasound machine using "S5-1" matrix array transducers (Philips Medical Systems, Andover, USA) equipped with STE technology, using a multi frequency (1- $5 \mathrm{MHz})$. ECGgated examination mostly used to help us during image acquisition \& later analysis. The images were digitally stored for off-line analysis (Q LAB version 9.0).
- Chamber quantification was performed in accordance with the recommendations of the American Society of Echocardiography and the European Association of Cardiovascular Imaging ${ }^{(9)}$.

\section{The following procedures were taken:}

\section{1) Aortic root (AR) ad Left atrium (LA) dimensions:}

The AR and LA dimensions were measured in the parasternal long-axis view perpendicular to the aortic root long axis, and measured at the level of the aortic sinuses by using the leading-edge to leading-edge convention ${ }^{(9)}$.

2) Interventricular septum dimension at end-diastole (IVSd), Left ventricular internal dimension at enddiastole (LVIDd), Left ventricular internal dimension at end-systole (LVIDs) and left ventricular posterior wall dimension (LVPWD): Linear internal measurements of the LV were acquired in the parasternal long-axis view carefully obtained perpendicular to the LV long axis, and measured at the level of the mitral valve leaflet tips ${ }^{(9)}$.

- Left ventricular end diastolic dimension (LVEDD), normal range (4.2-5.8 cm in males and 5.3-5.6 in females) - Left ventricular end systolic dimension (LVESD), normal range

(2.5-4.0 $\mathrm{cm}$ in males and $2.2-3.5 \mathrm{~cm}$ in females)

- Inter ventricular septal diameter (IVSD), normal range

(0.6- $1 \mathrm{~cm}$ in males and $0.6-0.9 \mathrm{~cm}$ in females)

- Left ventricular posterior wall diameter (LVPWD), normal range $(0.6-1 \mathrm{~cm}$ in males and $0.6-0.9 \mathrm{~cm}$ in females) ${ }^{(\mathbf{1 1})}$.

4) LV systolic function: by calculating Ejection Fraction (EF) using M-mode method: Measuring the dimension of left ventricle, from the leading edge of septal endocardial echo to the leading edge of posterior wall of endocardium distal to the tip of the mitral valve leaflets in the parasternal long axis view by using Teichholz's formula.

LV systolic function using modified Simpson's method:

Tracing of the blood-tissue interface in the apical fourand the apical two-chamber views. Then EF is calculated from EDV and ESV estimates, using the following modified Simpson's formula (Lang et al., 2015).

$\mathrm{EF}=(\mathrm{LVEDV}-\mathrm{LVESV}) /(\mathrm{LVEDV})$

5) LA diameter was measured in left parasternal long axis view at the level of aorta and left atrium at diastole. Left atrial (LA) volume in systole was also measured just before the mitral valve opening, using the biplane Simpson's method, as a mean between the values recorded in apical four- and two-chamber approaches. Subsequently, LAV was indexed for BSA, such as LAVI in $\mathrm{mL} / \mathrm{m} 2{ }^{(9)}$.

6) Several conventional parameters of $L V$ diastolic function were assessed. Transmitral E-wave velocity, Ewave deceleration time (DT), and late diastolic wave (A) velocity were measured applying pulse-wave Doppler (sample volume $=2 \mathrm{~mm}$ ) at the tip of the mitral leaflets in the 4-chamber view. 
7) The diastolic dysfunction grades was determined according to the criteria proposed by the European Association of Echocardiography:14 (i) normal diastolic function when $E^{\prime} \geq 9 \mathrm{~cm} / \mathrm{s}$ and LA volume $\leq 34 \mathrm{~mL} / \mathrm{m} 2$, (ii) mild diastolic dysfunction (grade I) when E/A ratio , 0.8 , DT $.200 \mathrm{~ms}$, and $\mathrm{E} / \mathrm{E}^{\prime}$ ratio $\leq 8$, (iii) moderate diastolic dysfunction (grade II) when E/A ratio 0.8-1.5, DT between 160-200 ms and $\mathrm{E} / \mathrm{E}^{\prime}$ ratio between 9 and 12, and (iv) severe diastolic dysfunction (grade III) when E/A ratio $\geq 2$, DT , $160 \mathrm{~ms}$, and $\mathrm{E} / \mathrm{E}^{\prime}$ ratio $\geq 13$ ).

8)TDI was recorded with high frame rate $(\geq 100$ frames/second) from the apical 4-chamber view to assess myocardial velocities. Peak annular early diastolic velocity ( $\left.\mathrm{E}^{\prime}\right)$ was measured in 2 annular LV segments (septal and lateral) and averaged to calculate the mean early diastolic velocity. The ratio $\mathrm{E} / \mathrm{E}^{\prime}$ was calculated, as a validated estimate of $L V$ filling pressure, and significant LV diastolic dysfunction was defined as $\mathrm{E}_{\mathrm{E}} \mathrm{E} \geq 15.0^{(\mathbf{1})}$.

9) speckle tracking Echocardiography:

Apical four- chamber views were obtained using conventional two-dimensional gray scale echocardiography, during breath hold with a stable ECG recording. Particular attention was given to obtain an adequate gray scale image, allowing reliable delineation of myocardial tissue and extracardiac structures. Three consecutive heart cycles were recorded and averaged. The frame rate is set between 60 and 80 frames per second; these settings are recommended to combine temporal resolution with adequate spatial definition, and to enhance the feasibility of the frame-to-frame tracking technique ${ }^{(\mathbf{1 2})}$.

In particular, Lt atrial endocardial surface is manually traced in four chamber view by a point-and-click approach. An epicardial surface tracing is then automatically generated by the system, thus creating a region of interest (ROI). To trace the ROI in the discontinuity of LA wall corresponding to pulmonary veins and LA appendage, the direction of LA endocardial and epicardial surfaces at the junction with these structures is extrapolated. After manual adjustment of ROI width and shape, the software divides the ROI into 6 segments with the possibility of further manual correction. Segments in which no adequate image quality can be obtained are rejected by the software and excluded from the analysis (11).

Lastly the software generates the longitudinal strain curves for each segment and a mean curve of all segments that reflect the pathophysiology of atrial function.

During the reservoir phase, the LA fills up, stretches itself, and for this reason, the atrial strain increases, reaching a positive peak at the end of atrial filling, before the opening of the mitral valve; after the opening of the mitral valve, LA empties quickly, shortens, the strain decreases, up to a plateau corresponding to the phase of diastasis, followed by a second positive peak, but less than the first, which corresponds to the period preceding the atrial contraction, and finally a negative peak after the atrial contraction. This second positive deflection of the atrial strain curve, corresponding to atrial systole, is present only if the subject analyzed presents sinus rhythm.

Thus, peak atrial longitudinal strain (PALS), measured at the end of the reservoir phase, and peak atrial contraction strain (PACS), measured just before the start of the active atrial contractile phase, are calculated by averaging values observed in all LA segments (global PALS and PACS), and by separately averaging values observed in four chamber view (four chamber average PALS and PACS, respectively ${ }^{(\mathbf{1 3})}$.

Regarding the measurement of peak atrial strain, as stated in the current ASE/EAE Consensus. Two techniques have been proposed to quantify atrial deformation by STE, which differ only by the choice of frame from which start processing software. The first takes as reference point the QRS onset and measures the positive peak atrial longitudinal strain, corresponding to atrial reservoir, the second uses the $\mathrm{P}$ wave as the reference point, enabling the measurement of a first negative peak atrial longitudinal strain, corresponding to atrial systole, a second positive peak atrial strain, corresponding to LA conduit function, and their sum ${ }^{(3)}$.

All data will be transferred to a workstation for further offline analysis. In blinded post-processing, global longitudinal deformation had been assessed by speckle tracking. Segments with poor image acquisition or artifacts were excluded due to inability to measure global longitudinal strain. We traced the endocardium of both atria simultaneously. A dedicated software for both atria strain analysis has not yet been released, so the analysis was performed using a software created for the left ventricle; for this reason, it was mandatory to be careful in the endocardial border delineation, excluding from the analysis the auricular and the outlet of the pulmonary veins, in order to minimize the risk of artifacts caused by signals from these structures ${ }^{(\mathbf{1 2})}$.

\section{Statistical analysis}

The recorded data were analyzed using the statistical package for social sciences, version 20.0 (SPSS Inc, Chicago, Illinois, USA). Quantitative data were expressed as mean \pm standard deviation (SD). Qualitative data were expressed as frequency and percentage.

\section{The following tests were done:}

- Independent-samples t-test of significance was used when comparing between two means.

- Chi-square $\left(\mathrm{x}^{2}\right)$ test of significance was used in order to compare proportions between two qualitative parameters.

- The confidence interval was set to $95 \%$ and the margin of error accepted was set to $5 \%$. The p-value was considered significant as the following:

- Probability (P-value)

- P-value <0.05 was considered significant.

- P-value $<0.001$ was considered as highly significant.

- P-value >0.05 was considered insignificant. 
RESULTS

Table (1): Comparison between the four groups revealed statistically non-significant differences in mean values of Sex.

\begin{tabular}{|c|c|c|c|c|c|c|}
\hline \multicolumn{2}{|l|}{ Sex } & $\overline{\text { G I }}$ & $\overline{\text { G II }}$ & G III & Control & G IV \\
\hline \multirow{2}{*}{ Male } & $\mathbf{N}$ & 9 & 9 & 12 & 11 & 41 \\
\hline & $\%$ & $45.0 \%$ & $45.0 \%$ & $60.0 \%$ & $55.0 \%$ & $51.3 \%$ \\
\hline \multirow{2}{*}{ Female } & $\mathbf{N}$ & 11 & 11 & 8 & 9 & 39 \\
\hline & $\%$ & $55.0 \%$ & $55.0 \%$ & $40.0 \%$ & $45.0 \%$ & $48.8 \%$ \\
\hline \multirow{2}{*}{ Total } & $\mathbf{N}$ & 20 & 20 & 20 & 20 & 80 \\
\hline & $\%$ & $100.0 \%$ & $100.0 \%$ & $100.0 \%$ & $100.0 \%$ & $100.0 \%$ \\
\hline \multirow{2}{*}{ Chi-square } & $\mathbf{X}^{2}$ & \multicolumn{5}{|l|}{1.351} \\
\hline & P-value & \multicolumn{5}{|l|}{0.717} \\
\hline
\end{tabular}

There was statistically non-significant difference in mean values of Sex , P-value( 0.71)

Table (2): Comparison between the four groups revealed statistically non-significant differences in mean values of HTN.

\begin{tabular}{|c|c|c|c|c|c|c|}
\hline \multicolumn{2}{|l|}{ HTN } & G I & G II & G III & Control & G IV \\
\hline \multirow{2}{*}{ Yes } & $\mathbf{N}$ & 7 & 13 & 13 & 7 & 40 \\
\hline & $\%$ & $35.0 \%$ & $65.0 \%$ & $65.0 \%$ & $35.0 \%$ & $50.0 \%$ \\
\hline \multirow{2}{*}{ No } & $\mathbf{N}$ & 13 & 7 & 7 & 13 & 40 \\
\hline & $\%$ & $65.0 \%$ & $35.0 \%$ & $35.0 \%$ & $65.0 \%$ & $50.0 \%$ \\
\hline \multirow{2}{*}{ Total } & $\mathbf{N}$ & 20 & 20 & 20 & 20 & 80 \\
\hline & $\%$ & $100.0 \%$ & $100.0 \%$ & $100.0 \%$ & $100.0 \%$ & $100.0 \%$ \\
\hline \multirow{2}{*}{ Chi-square } & $\mathbf{X}^{2}$ & \multicolumn{5}{|l|}{7.200} \\
\hline & P-value & \multicolumn{5}{|l|}{0.066} \\
\hline
\end{tabular}

There was statistically non-significant difference in mean values of HTN, P-value( 0.06)

Table (3): Comparison between the four groups revealed statistically non-significant differences in mean values of DM

\begin{tabular}{|c|c|c|c|c|c|c|}
\hline \multicolumn{2}{|l|}{ DM } & G I & G II & G III & Control & G IV \\
\hline \multirow{2}{*}{ Yes } & $\mathbf{N}$ & 6 & 6 & 5 & 3 & 20 \\
\hline & $\%$ & $30.0 \%$ & $30.0 \%$ & $25.0 \%$ & $15.0 \%$ & $25.0 \%$ \\
\hline \multirow{2}{*}{ No } & $\mathbf{N}$ & 14 & 14 & 15 & 17 & 60 \\
\hline & $\%$ & $70.0 \%$ & $70.0 \%$ & $75.0 \%$ & $85.0 \%$ & $75.0 \%$ \\
\hline \multirow{2}{*}{ Total } & $\mathbf{N}$ & 20 & 20 & 20 & 20 & 80 \\
\hline & $\%$ & $100.0 \%$ & $100.0 \%$ & $100.0 \%$ & $100.0 \%$ & $100.0 \%$ \\
\hline \multirow{2}{*}{ Chi-square } & $\mathbf{X}^{2}$ & \multicolumn{5}{|l|}{1.600} \\
\hline & P-value & \multicolumn{5}{|l|}{0.659} \\
\hline
\end{tabular}

There was statistically non-significant difference in mean values of DM, P-value( 0.65$)$ 
Table (4): Comparison between the four groups revealed statistically non-significant differences in mean values of Age, DBP, HR and BMI.

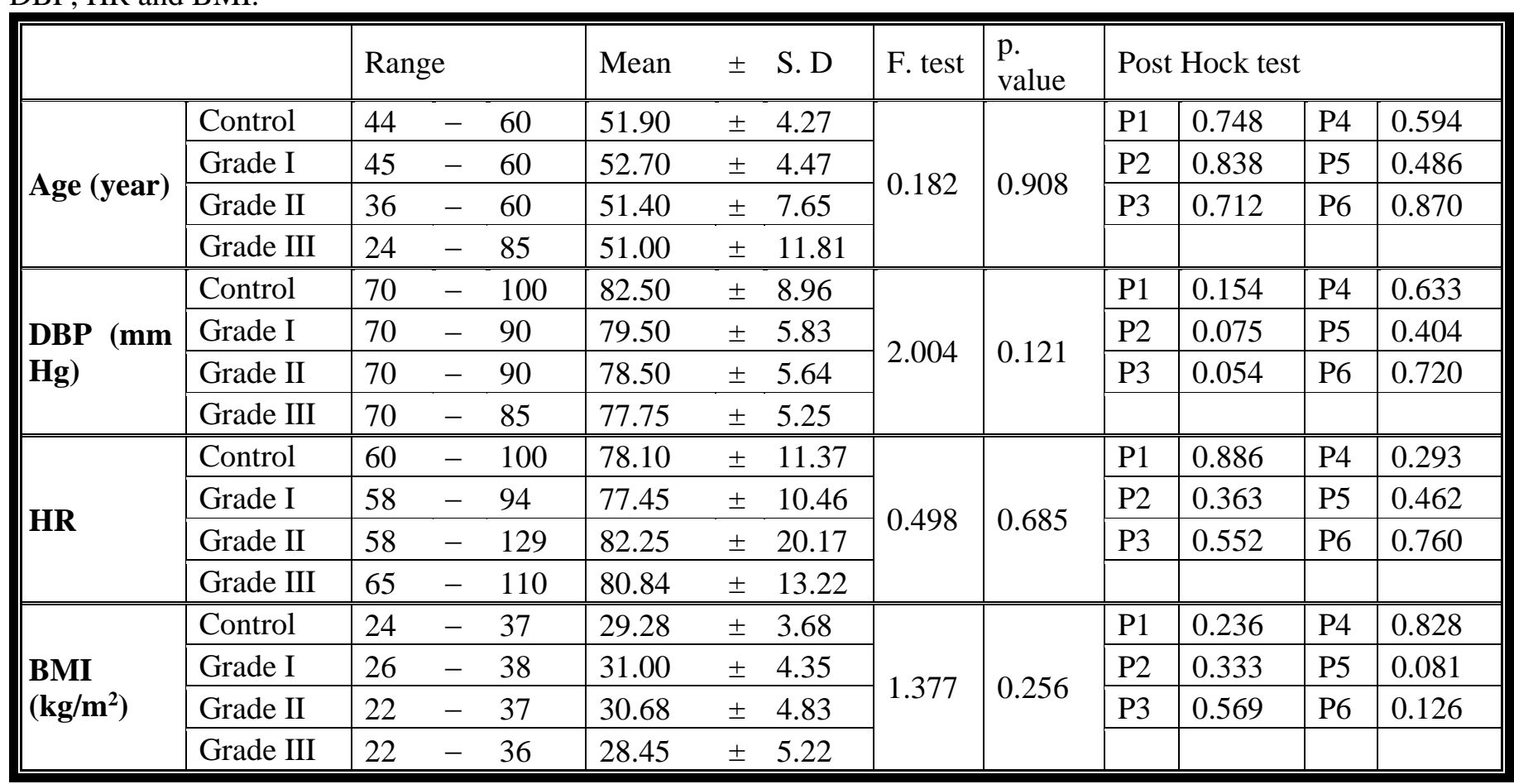

There were statistically non-significant difference in mean values of Age, DBP, HR and BMI. , P-value(0.908, $0.121,0.685,0.256)$ consequently.

Table (5): Comparison between the four groups revealed statistically significant difference in mean values of SBP

\begin{tabular}{|c|c|c|c|c|c|c|c|c|c|c|c|c|}
\hline & & \multicolumn{3}{|c|}{ Range } & \multirow{2}{*}{$\begin{array}{l}\text { Mean } \\
134.00\end{array}$} & \multirow{2}{*}{$\begin{array}{l} \pm \text { S. D } \\
\pm 18.61\end{array}$} & $\begin{array}{l}\text { F. } \\
\text { test }\end{array}$ & $\begin{array}{l}\mathrm{p} . \\
\text { value }\end{array}$ & \multicolumn{4}{|c|}{ Post Hock test } \\
\hline \multirow{4}{*}{$\begin{array}{l}\text { SBP } \\
(\mathrm{mm} \mathrm{Hg})\end{array}$} & Control & 110 & - & 170 & & & \multirow{4}{*}{4.172} & \multirow{4}{*}{$0.009 *$} & $\mathrm{P} 1$ & $0.01 *$ & $\mathrm{P} 4$ & 0.529 \\
\hline & Grade I & 110 & - & 160 & 124.50 & \pm 11.57 & & & $\mathrm{P} 2$ & $0.003 *$ & $\mathrm{P} 5$ & 0.529 \\
\hline & Grade II & 110 & - & 135 & 122.00 & \pm 8.49 & & & P3 & $0.003 *$ & P6 & 1.0 \\
\hline & Grade III & 110 & - & 135 & 122.00 & \pm 8.49 & & & & & & \\
\hline
\end{tabular}

There was statistically significant difference in mean values of SBP , P-value(0.009) 
Table (6): Comparison between the four groups revealed statistically significant difference in mean values of Echocardiographic data.

\begin{tabular}{|c|c|c|c|c|c|c|c|c|c|c|c|c|}
\hline \multirow{5}{*}{\begin{tabular}{|l} 
\\
LVEDD
\end{tabular}} & \multirow{3}{*}{$\begin{array}{l}\text { Control } \\
\text { Grade I } \\
\end{array}$} & \multicolumn{3}{|c|}{ Range } & \multirow{2}{*}{$\begin{array}{l}\text { Mean } \\
4.85 \\
\end{array}$} & \multirow{2}{*}{$\begin{array}{l} \pm \text { S. D } \\
\pm 0.24 \\
\end{array}$} & \multirow{5}{*}{$\begin{array}{l}\text { F. test } \\
3.822\end{array}$} & \multirow{5}{*}{$\begin{array}{l}\text { p. value } \\
0.013^{*}\end{array}$} & \multicolumn{4}{|c|}{ Post Hock test } \\
\hline & & 4.44 & - & 5.28 & & & & & \multicolumn{4}{|c|}{\begin{tabular}{l|l} 
P1 & 0.971
\end{tabular}} \\
\hline & & 4.46 & - & 5.31 & 4.85 & \pm 0.26 & & & $\mathrm{P} 2$ & 0.287 & P5 & $0.005^{*}$ \\
\hline & Grade II & 3.8 & - & 5.35 & 4.72 & \pm 0.40 & & & P3 & $0.004 *$ & P6 & 0.064 \\
\hline & Grade III & 3.79 & - & 5.56 & 4.49 & \pm 0.57 & & & & & & \\
\hline \multirow{4}{*}{ LVESD } & Control & 2.97 & - & 3.48 & 3.25 & \pm 0.16 & \multirow{4}{*}{6.777} & \multirow{4}{*}{$0.001 *$} & P1 & 0.729 & P4 & 0.253 \\
\hline & Grade I & 3.03 & - & 4.41 & 3.30 & \pm 0.30 & & & $\mathrm{P} 2$ & 0.424 & P5 & $0.001 *$ \\
\hline & Grade II & 2.02 & - & 4.03 & 3.16 & \pm 0.41 & & & $\mathrm{P} 3$ & $0.001 *$ & P6 & $0.005^{*}$ \\
\hline & Grade III & 2.04 & - & 3.86 & 2.81 & \pm 0.54 & & & & & & \\
\hline \multirow{4}{*}{$\operatorname{LVFS}(\%)$} & Control & 30.6 & - & 35.7 & 32.61 & \pm 1.30 & \multirow{4}{*}{9.381} & \multirow{4}{*}{$0.001 *$} & P1 & 0.752 & $\mathrm{P} 4$ & 0.209 \\
\hline & Grade I & 30.3 & - & 35.3 & 32.27 & \pm 1.24 & & & $\mathrm{P} 2$ & 0.345 & P5 & $0.001 *$ \\
\hline & Grade II & 30.1 & - & 41.1 & 33.65 & \pm 3.03 & & & P3 & $0.001 *$ & P6 & $0.001 *$ \\
\hline & Grade III & 29 & - & 45.9 & 37.41 & \pm 5.92 & & & & & & \\
\hline & Control & 58.6 & - & 65.2 & 61.00 & \pm 1.60 & & & P1 & 0.916 & P4 & 0.246 \\
\hline & Grade I & 58.7 & - & 64.5 & 60.87 & \pm 1.37 & & & $\mathrm{P} 2$ & 0.291 & P5 & $0.001 *$ \\
\hline LVEF(\%) & Grade II & 57.7 & - & 71.1 & 62.26 & \pm 3.59 & 8.806 & $0.001^{*}$ & P3 & $0.001 *$ & P6 & $0.001 *$ \\
\hline & Grade III & 56 & - & 77.6 & 66.19 & \pm 6.23 & & & & & & \\
\hline & Control & 13 & - & 37 & 26.49 & \pm 7.00 & & & $\mathrm{P} 1$ & 0.387 & $\mathrm{P} 4$ & $0.001 *$ \\
\hline LA volume & Grade I & 19 & - & 41 & 28.91 & \pm 6.72 & & & $\mathrm{P} 2$ & $0.001 *$ & P5 & $0.001 *$ \\
\hline$(\mathbf{m l} / \mathbf{m} 2)$ & Grade II & 35 & - & 61 & 48.25 & \pm 8.82 & 45.251 & $0.001^{*}$ & P3 & $0.001 *$ & P6 & 0.135 \\
\hline & Grade III & 33 & - & 68 & 52.45 & \pm 11.72 & & & & & & \\
\hline & Control & 78 & - & 102 & 90.64 & \pm 7.98 & & & P1 & $0.001 *$ & P4 & $0.001^{*}$ \\
\hline & Grade I & 52 & - & 82 & 68.76 & \pm 10.51 & $51 \quad 104$ & $0001 *$ & $\mathrm{P} 2$ & $0.001 *$ & P5 & $0.001 *$ \\
\hline $\mathrm{E}(\mathrm{cm} / \mathrm{s})$ & Grade II & 88 & - & 132 & 111.45 & \pm 15.15 & 51.104 & 0.001 & P3 & $0.001 *$ & P6 & 0.443 \\
\hline & Grade III & 89 & - & 140 & 114.70 & \pm 17.47 & & & & & & \\
\hline & Control & 55.6 & - & 100 & 72.67 & \pm 13.68 & & & P1 & $0.001 *$ & $\mathrm{P} 4$ & $0.001 *$ \\
\hline & Grade I & 70.3 & - & 136 & 102.62 & \pm 20.57 & 46760 & $0001 *$ & $\mathrm{P} 2$ & 0.060 & P5 & $0.001 *$ \\
\hline $\mathbf{A}(\mathbf{c m} / \mathbf{s})$ & Grade II & 56.4 & - & 120 & 81.96 & \pm 16.42 & 40.100 & $0.001 *$ & P3 & $0.001 *$ & P6 & $0.001 *$ \\
\hline & Grade III & 33.8 & - & 60.86 & 46.01 & \pm 7.97 & & & & & & \\
\hline & Control & 1 & - & 1.6 & 1.28 & $\begin{array}{l} \pm 0.18 \\
\end{array}$ & & & P1 & 0.001* & P4 & $0.001^{*}$ \\
\hline F/A notio & Grade I & 0.6 & - & 0.76 & 0.68 & \pm 0.06 & 274610 & $0001 *$ & $\mathrm{P} 2$ & 0.082 & P5 & $0.001 *$ \\
\hline E/A ratio & Grade II & 1 & - & 1.9 & 1.39 & \pm 0.23 & $2 / 4.610$ & $0.001 *$ & P3 & $0.001 *$ & P6 & $0.001 *$ \\
\hline & Grade III & 2 & - & 3.1 & 2.52 & \pm 0.28 & & & & & & \\
\hline & Control & 8 & - & 10 & 9.03 & \pm 0.66 & & & P1 & 0.024* & P4 & 0.889 \\
\hline & Grade I & 4 & - & 9 & 5.48 & \pm 1.00 & 2262 & 0088 & $\mathrm{P} 2$ & $0.033^{*}$ & P5 & 0.297 \\
\hline Septal E & Grade II & 5 & - & 6.5 & 5.70 & \pm 0.43 & 2.202 & 0.088 & P3 & 0.212 & P6 & 0.365 \\
\hline & Grade III & 4.1 & - & 48 & 7.10 & \pm 9.64 & & & & & & \\
\hline & Control & 1.8 & - & 14 & 11.72 & \pm 2.60 & & & $\mathrm{P} 1$ & $0.001 *$ & $\mathrm{P} 4$ & 0.356 \\
\hline T' & Grade I & 6.2 & - & 8 & 7.14 & \pm 0.54 & 18260 & $\cap \Omega 01 *$ & $\mathrm{P} 2$ & $0.001 *$ & P5 & 0.482 \\
\hline Lateral E & Grade II & 5 & - & 9 & 6.68 & \pm 1.23 & 48.200 & $0.001 *$ & P3 & $0.001 *$ & P6 & 0.825 \\
\hline & Grade III & 5 & - & 9 & 6.79 & \pm 1.13 & & & & & & \\
\hline & Control & 27 & - & 223 & 210.35 & \pm 43.21 & & & P1 & $0.001 *$ & P4 & 0.001* \\
\hline & Grade I & 230 & - & 238 & 233.80 & \pm 2.78 & 20871 & & $\mathrm{P} 2$ & 0.088 & P5 & $0.001 *$ \\
\hline DT (ms) & Grade II & 190 & - & 200 & 198.47 & \pm 2.10 & $29.8 / 1$ & $0.001^{*}$ & P3 & $0.001 *$ & P6 & $0.001 *$ \\
\hline & Grade III & 166 & - & 174 & 170.00 & \pm 2.43 & & & & & & \\
\hline & Control & 7.4 & - & 11 & 8.87 & \pm 1.01 & & & P1 & $0.001 *$ & $\mathrm{P} 4$ & $0.001 *$ \\
\hline F/F Moon & Grade I & 1.6 & - & 16 & 13.18 & \pm 3.10 & 102385 & $0001 *$ & $\mathrm{P} 2$ & $0.001 *$ & P5 & $0.001 *$ \\
\hline E/E Mean & Grade II & 15.8 & - & 23 & 19.76 & \pm 2.15 & 102.385 & 0.001 & $\mathrm{P} 3$ & $0.001 *$ & P6 & $0.003^{*}$ \\
\hline & Grade III & 7.9 & - & 26 & 22.42 & \pm 3.81 & & & & & & \\
\hline & Control & 15 & - & 30 & 20.80 & \pm 4.74 & & & P1 & 0.001* & P4 & (0.001* \\
\hline & Grade I & 28 & - & 37 & 32.28 & \pm 3.13 & & & $\mathrm{P} 2$ & $0.001 *$ & P5 & $0.001 *$ \\
\hline TR veloty & Grade II & 27 & - & 52 & 39.25 & \pm 7.57 & 44.794 & $0.001^{*}$ & P3 & $0.001 *$ & P6 & 0.083 \\
\hline & Grade III & 29 & - & 58 & 42.85 & \pm 8.88 & & & & & & \\
\hline
\end{tabular}


Table (7): Comparison between the four groups revealed statistically significant difference in mean values of LA speckle tracking.

\begin{tabular}{|c|c|c|c|c|c|c|c|c|c|c|c|c|}
\hline & & Ran & & & Mean & \pm S.D & F. test & p. value & Pos & Hock te & & \\
\hline \multirow{4}{*}{ GLS } & Control & 28 & - & 64 & 45.70 & \pm 10.58 & \multirow{4}{*}{26.986} & \multirow{4}{*}{$0.001 *$} & P1 & $0.001^{*}$ & $\mathrm{P} 4$ & $0.019 *$ \\
\hline & Grade I & 24 & - & 55 & 35.59 & \pm 9.03 & & & $\mathrm{P} 2$ & $0.001^{*}$ & $\mathrm{P} 5$ & $0.001 *$ \\
\hline & Grade II & 18 & - & 44 & 28.96 & \pm 7.95 & & & P3 & $0.001 *$ & $\mathrm{P} 6$ & $0.011 *$ \\
\hline & Grade III & 9.4 & - & 33.2 & 21.77 & \pm 7.08 & & & & & & \\
\hline \multirow{4}{*}{$\begin{array}{l}\text { Peak LA } \\
(\%)\end{array}$} & Control & 24 & - & 499 & 35.40 & \pm 7.58 & \multirow{4}{*}{57.479} & \multirow{4}{*}{$0.001 *$} & P1 & $0.001^{*}$ & P4 & $0.001^{*}$ \\
\hline & Grade I & 21 & - & 37 & 28.20 & \pm 5.37 & & & $\mathrm{P} 2$ & $0.001^{*}$ & $\mathrm{P} 5$ & $0.001 *$ \\
\hline & Grade II & 13 & - & 30 & 20.20 & \pm 6.02 & & & P3 & $0.001^{*}$ & $\mathrm{P} 6$ & $0.001 *$ \\
\hline & Grade III & 7 & - & 24 & 11.95 & \pm 4.47 & & & & & & \\
\hline
\end{tabular}

There were statistically significant difference in mean values of GLS , Peak LA (\%) ; P-value $(0.001,0.001)$
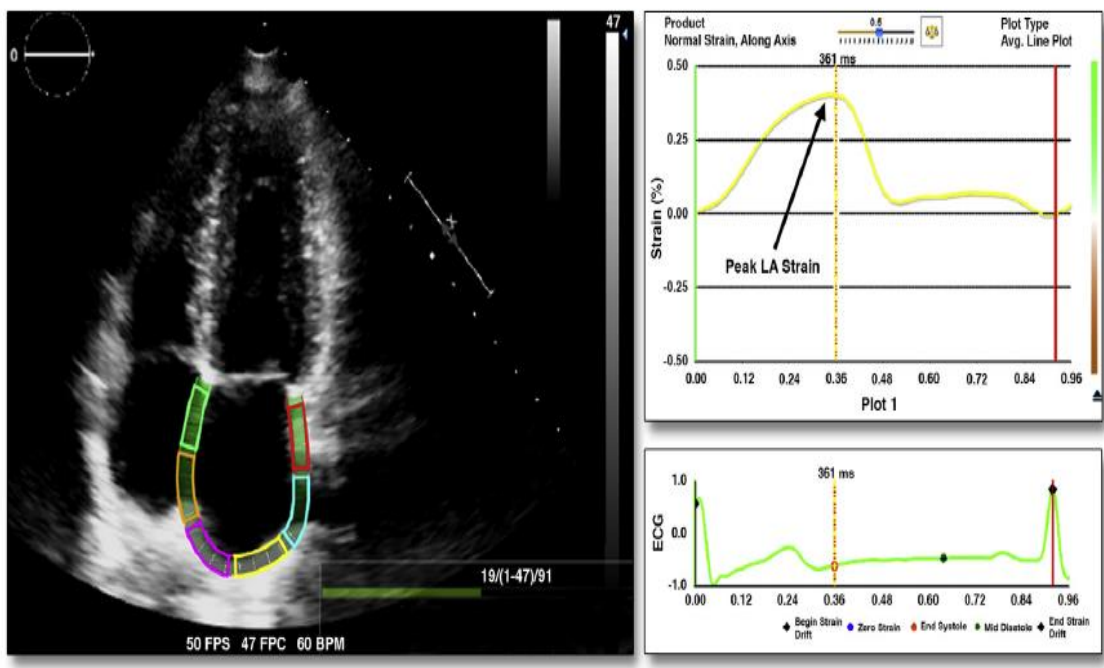

Figure (1): The apical 4-chamber view with the entirety of the left atrium (LA) is pictured, with the endocardium of the LA traced (left). LA strain over time curve and an electrocardiogram signal are shown on the right.

\section{DISCUSSION}

We clinically evaluated 80 subjects who were classified into control group in addition to three patient groups with different grades of diastolic dysfunction. These groups were Group 1: (control group): includes 20 patients with normal echocardiography which represented 25\% of studied patients, Group 2: Grade I diastolic dysfunction (20) patients. Group 3: Grade II diastolic dysfunction (20) patients and Group 4: Grade IV diastolic dysfunction (20) patients.

They were subjected to detailed echocardiographic examinations of the LV systolic and diastolic function and Lt atrial strain by 2D speckle tracking.

The results of the current work show that there was statistically significant difference between the control and other three groups as regard peak LA strain.

This is in conformity with the findings of Singh $\boldsymbol{e t}$ al. ${ }^{(14)}$ who investigated 90 subjects included 15 patients with normal diastolic function, and 3 subgroups of 25 subjects with grades 1,2, and 3 of DD and found statistical significance between the two study groups.

This is also in agreement with the findings of Singh et al. ${ }^{(14)}$ who studied 76 patients, involved a retrospective derivation group $(\mathrm{N}=26)$ and an independent prospective validation cohort $(\mathrm{N}=50)$ to derive and then test a peak LA strain who demonstrated a great statistical significance between control and patients.
This is also in agreement with the findings of Morris et al. ${ }^{\left({ }^{(15)}\right.}$ who studied 517 patients in sinus rhythm at risk for LVDD such as those with arterial hypertension, diabetes mellitus, or history of coronary artery disease and preserved LVEF and analyze the potential usefulness and clinical relevance of adding left atrial (LA) strain to left atrial volume index (LAVI) in the detection of left ventricular diastolic dysfunction and found that In patients with LV diastolic alterations and estimated elevated LV filling pressures, the rate of abnormal LA strain was significantly higher.

There was statistically significant difference between the control and the other three groups as regard GLS of LA. These findings were also in agreements to Khan et al. ${ }^{(16)}$ who applied STI to 50 patient s with Grade 1-2 DD, comparing these results to 100 normal controls by which Global LA strain was significantly lower in comparison with the control group.

These findings were also in agreements to Hennawy et al. ${ }^{(10)}$ who studied 50 hypertensive patients and 50 age matched normotensive controls, all with normal LA volume index and free from any other cardiovascular disease that may affect the LA size or function and found that different indices of LA dysfunction (Total LA stroke volume, LA expansion index and global PALS) were significantly lower in the hypertensive group despite the normal LA volume index in all the studied subjects. The presence of diabetes 
mellitus (DM) and higher grade of LV diastolic dysfunction were significantly associated with lower global PALS. The higher age, systolic blood pressure (BP), body mass index (BMI), LA volume index, and LV mass index and the lower LA expansion index were associated with lower global PALS.

These findings were also in agreements to Cameli et al. ${ }^{(11)}$ who enrolled 162 patients affected by $\mathrm{AH}$ and/or DM with normal indexed LA volume, LV end-diastolic diameter and a LVEF > 52\% (females) or > 54\% (males) (60 hypertensive, 52 diabetics and 50 both) and 60 healthy controls and found that PALS and GAVS were significantly reduced in AH $(31.9 \pm 10.3 \%$ and $49.7 \pm$ $11.2 \%$, respectively) and DM (26.2 $\pm 7.1 \%$ and $42.6 \pm$ 9.8\%) compared to controls, and even more if the two coexisted $(20.4 \pm 6.5 \%$ and $37.1 \pm 8.4 \%)$. PALS had the highest statistical significance and were able to identify subclinical damage independently from LS value. PALS was reduced in patients with $\mathrm{AH}$ and/or DM without alteration of standard echo indexes. The value of PALS was independent from LS and was sufficient to identify heart dysfunction in an earlier stage.

There was statistically significant difference between the groups (Control and DD) in all echocardiographic parameters including LVEDD, LVESD, LVFS(\%), LVEF(\%), LA volume, E, A, E/A, Septal E', Lateral E', DT, E/E’ Mean, TR velocity .

This in agreement with the findings of Fang et al. ${ }^{(17)}$ who studied 177 consecutive patients with MetS (75 males with a mean age of $53.7 \pm 8.5$ years, range 26-74), with cardiovascular risk factors (obesity, hypertension, glucose tolerance/diabetes mellitus and dyslipidemia) and no previous history or clinical evidence of heart failure or overt coronary artery disease and also enrolled 156 ageand sex-matched normal subjects (57 males with a mean age of $52.7 \pm 8.7$ years, range $35-85$ years) without cardiovascular disease, hypertension or diabetes mellitus and found that the MetS patients showed decreased early diastolic peak tissue velocity of the mitral valve annulus (Veglobal), a lower ratio of early to late diastolic peak tissue velocity (Veglobal/Vaglobal) and a higher $\mathrm{E} /$ Veglobal ratio reflecting LV diastolic function.

\section{CONCLUSION}

This study was designed to observe the relationship between left atrial (LA) strain and left ventricular diastolic function. By Two-dimensional (2D) speckle tracking echocardiography parameters of LA, there was variation in different groups of diastolic dysfunction in comparison to control group. LA strain could be used to detect diastolic dysfunction (DD) and classify its degree when present.

\section{REFERENCES}

1. Kuwaki H, Takeuchi M, Chien-Chia Wu V et al. (2014): Redefining diastolic dysfunction grading: combination of $\mathrm{E} / \mathrm{A} \# 0.75$ and deceleration time $>140 \mathrm{~ms}$ and $\mathrm{E} / \varepsilon 0 \$ 10$. J AmColl Cardiol Img., 7:749-58.
2. Cameli M, Caputo M, Mondillo S et al. (2009): Feasibility and reference values of left atrial longitudinal strain imaging by two-dimensional speckle tracking. Cardiovasc Ultrasound, 7:6-11.

3. Saraiva RM, Demirkol S, Buakhamsri A et al. (2010): Left atrial strain measured by two dimensional speckle tracking represents a new tool to evaluate left atrial function. J Am Soc Echocardiogr., 23:172-80.

4. Vianna-Pinton R, Moreno CA, Baxter CM et al. (2009): Two-dimensional speckle-tracking echocardiography of the left atrium: feasibility and regional contraction and relaxation differences in normal subjects. J Am Soc Echocardiogr., 22:299-305.

5. Mondillo S, Cameli M, Caputo ML et al. (2011): Early detection of left atrial strain abnormalities by speckletracking in hypertensive and diabetic patients with normal left atrial size. J Am Soc Echocardiogr., 24:898-908.

6. Inaba Y, Yuda S, Kobayashi N et al. (2005): Strain rate imaging for noninvasive functional quantification of the left atrium: comparative studies in controls and patients with atrial fibrillation. J Am Soc Echocardiogr., 18:729-36

7. Kurt M, Wang J, Torre-Amione G et al. (2009): Left atrial function in diastolic heart failure. Circ Cardiovasc Imaging, 2:10-5.

8. Lang RM, Badano LP, Mor-Avi V et al. (2015): Recommendations for cardiac chamber quantification by echocardiography in adults: an update from the American Society ofn Echocardiography and the European Association of Cardiovascular Imaging. J Am Soc Echocardiogr., 28:1-39.

9. Melenovsky V, Hwang SJ, Redfield MM et al. (2015): Left atrial remodeling and function in advanced heart failure with preserved or reduced ejection fraction. Circ Heart Fail., 8:295-303.

10. Hennawy B, El Kilany W, Galal H et al. (2018): Role of speckle tracking echocardiography in detecting early left atrial dysfunction in hypertensive patients. Egyptian Heart Journal, 70(3): 217-223.

11. Cameli M, Sparla S, Losito $M$ et al. (2016): Correlation of left atrial strain and Doppler measurements with invasive measurement of left ventricular enddiastolic pressure in patients stratified for different values of ejection fraction. Echocardiography, 33:398-405.

12. Liu Y, Wang K, Su D et al. (2014): Noninvasive assessment of left atrial phasic function in patients with hypertension and diabetes using twodimensional speckle tracking and volumetric parameters. Echocardiography, 31:727-35.

13. Amita Singh, Karima Addetia, Francesco Maffessanti et al. LA Strain for Categorization of LV Diastolic Dysfunction j.jcmg.2016.08.014

14. Singh A, Medvedofsky D, Mediratta A et al. (2019): Peak left atrial strain as a single measure for the non-invasive assessment of left ventricular filing pressures. Int $\mathbf{J}$ Cardiovasc Imaging, 35(1): 23-32.

15. Morris DA, Takeuchi M, Krisper M et al. (2015): Normal values and clinical relevance of left atrial myocardial function analysed by speckle-tracking echocardiography multicentre study. Eur Heart J Cardiovasc Imaging, 16:364-72.

16. Khan UA, de Simone G, Hill J et al. (2013): Depressed Atrial Function in Diastolic Dysfunction: A Speckle Tracking Imaging Study. Echocardiography, 30(3):309-16. Fang N, Sui D, Yu J et al. (2015): Strain/strain rate imaging of impaired left atrial function in patients with metabolic syndrome. Hypertens Res., 38(11):758-64. 